\title{
Anti-inflammatory diet and inflammatory bowel disease: what clinicians and patients should know?
}

\author{
Nor Hamizah Shafiee ${ }^{1}$, Zahara Abdul Manaf', Norfilza M. Mokhtar, ${ }^{3,4}$, Raja Affendi Raja Ali ${ }^{4,5}$ \\ ${ }^{1}$ Department of Medicine, Faculty of Medicine, ${ }^{2}$ Dietetics Programme, Faculty of Health Sciences, ${ }^{3}$ Department of Physiology, Faculty of \\ Medicine, and ${ }^{4}$ GUT Research Group, Faculty of Medicine, Universiti Kebangsaan Malaysia, Kuala Lumpur; ${ }^{5}$ Gastroenterology Unit, \\ Department of Medicine, UKM Medical Centre, Kuala Lumpur, Malaysia
}

Current treatment for inflammatory bowel disease (IBD) includes the application of anti-inflammatory agents for the induction and remission of IBD. However, prolonged use of anti-inflammatory agents can exert adverse effects on patients. Recently, formulated dietary approach in treating IBD patients is utilized to improve clinical activity scores. An alteration of gastrointestinal microbiota through dietary therapy was found to reduce IBD and is recognized as a promising therapeutic strategy for IBD. One of the recommended formulated diets is an anti-inflammatory diet (AID) that restricts the intake of carbohydrates with modified fatty acids. This diet also contains probiotics and prebiotics that can promote balanced intestinal microbiota composition. However, scientific evidences are limited to support this specific dietary regime in maintaining the remission and prevention relapse of IBD. Therefore, this review aimed to summarize available data from various studies to evaluate the AID diet effectiveness which will be useful for clinicians to manage their IBD patients by application of improved dietary therapy. (Intest Res 2021;19:171-185)

Key Words: Anti-inflammatory diet; Inflammatory bowel disease; Remission; Relapse; Nutritional intake

\section{INTRODUCTION}

Inflammatory bowel disease (IBD) is a chronic inflammatory disorder of gastrointestinal tract which includes Crohn's disease (CD) and ulcerative colitis (UC). For both CD and UC, patients usually experience periods of remission and a flareup of the disease. ${ }^{1}$ Currently, there is no cure for IBD, ${ }^{2}$ and available treatment approaches target to reduce inflammation and prevent clinical symptoms and complications. ${ }^{3}$ In the United States, the disparity in treatment goals was observed between the clinicians and patients, where $25 \%$ of clinicians versus $2.5 \%$ of patients viewed the treatment is successful as reflected by endoscopic remission. ${ }^{4}$

Received April 25, 2020. Revised December 3, 2020

Accepted December 3, 2020.

Correspondence to Raja Affendi Raja Ali, UKM Medical Centre, Universiti

Kebangsaan Malaysia, Jalan Yaacob Latif, Bandar Tun Razak, Cheras, Kuala Lumpur 56000, Malaysia. Tel: +60-3-91456094, Fax: +60-3-91456692,

E-mail:draffendi@ppukm.ukm.edu.my
Current treatments mostly involve the use of anti-inflammatory agents including corticosteroids, immunomodulators, and biological agents. ${ }^{5}$ However, drug therapy leads to inconsistent health improvement and severe adverse effects. ${ }^{6}$ Hence, manipulation of gut microbiota through diet change is a promising therapeutic strategy. This strategy can reduce inflammation by maintaining a healthy gut microbiota balance while optimizing good nutrition. ${ }^{7}$ The most well-studied dietary therapy for IBD is the use of exclusive enteral nutrition (EEN) with elemental, semi-elemental, and defined formula diets. ${ }^{8}$ The EEN is recommended as the first-line therapy for active CD patients as it can change the gut microbiota profile and promote mucosal healing through anti-inflammatory effects.

However, EEN have limitations in sustaining a long-term maintenance therapy for $\mathrm{IBD}^{10}$ and the liquid nutrition formula lacks palatability. ${ }^{11}$ The limitations led to the introduction of various oral formulated diets for IBD treatment. Even though 
patients seek dietary consultation with clinicians, whole food diet-based therapies for CD and UC are poorly developed. As previous studies showed that alteration of gut microbiota composition plays a significant role in the pathogenesis of IBD, ${ }^{12,13}$ formulated diet known as anti-inflammatory diet (AID) is of potential utilization. The IBD-AID was developed based on the relationship established between gut microbiota imbalance and intake of specific carbohydrates acting as substrates for pathogenic bacteria in the gut. ${ }^{14}$ The AID diet was reported to limit carbohydrates such as refined sugar, glutenbased grains, and processed complex carbohydrates that can enhance the growth of inflammatory bacteria in the digestive tract. $^{14}$

Essentially, IBD-AID consisted of 5 basic components: (1) consumption restriction of specific carbohydrates (e.g., lactose, refined or processed complex carbohydrates); (2) ingestion of prebiotics, probiotics and foods that are rich in components that can restore the balance of intestinal microbiota (e.g., soluble fiber, leek, onions, fermented foods); (3) reduced intake of total fat and saturated fatty acids and increased intake of omega-3 rich foods; (4) review of overall dietary pattern, identification of patient's intolerance and detection of any lacking nutrients; or (5) modification of food texture (e.g., cooked, homogenized, ground) to improve nutrient absorption in the gut. ${ }^{14}$ Table 1 demonstrates the complexity of texture modification of foods depending on the severity of symptoms and patient's tolerance. At the initial stage, soft foods should be introduced followed by well-cooked foods and can gradually revert to whole foods as the symptoms improve.

In the present review, the potential roles of AID as adjunctive therapy for IBD efficient remission therapy. With increasing patients' interest in AID, it is imperative to understand the effectiveness and limitation of this dietary intervention besides providing information for clinicians to consult their patients through improved dietary therapy.

\section{EFFECTIVENESS OF AID IN THE MANAGEMENT OF IBD}

Several studies showed anti-inflammatory foods can be beneficial and/or detrimental in maintaining the remission and prevention flares of IBD. In this review, information on AID that include prebiotics and probiotics, omega-3 rich foods, refined carbohydrates, gluten-based foods, lactose-based foods, high-fat foods, low-fermentable oligosaccharide, disaccharide, monosaccharide, and polyol (FODMAP) diet, and vita-

Table 1. Phases of the IBD-AID

\begin{tabular}{|c|c|c|}
\hline Phase & Why should I be following this phase? & Examples of foods \\
\hline I. Soft foods, pureed foods, no seeds & $\begin{array}{l}\text { Currently experiencing a flare, any bleeding, urgency } \\
\text { and high frequency of bowel movements or pain. This } \\
\text { phase is helpful for patients who have recently been } \\
\text { hospitalized. At this stage, you may not be able to } \\
\text { tolerate many foods, in part due to the texture of the } \\
\text { food. This phase emphasizes soft and pureed foods } \\
\text { using a blender. Tolerance of foods are individualized. }\end{array}$ & $\begin{array}{l}\text { Smoothies, well-cooked whole (groats) or steel } \\
\text { cut oats, ground flax or chia seeds (if you can } \\
\text { tolerate ground seeds) pureed soups, pureed } \\
\text { vegetables, yogurt and miso (good sources of } \\
\text { probiotics), and ground lean meats. }\end{array}$ \\
\hline $\begin{array}{l}\text { II. Soft textures, well-cooked, no } \\
\text { seeds. May still need to avoid stems, } \\
\text { choose floppy greens or other greens } \\
\text { depending on individual tolerance. }\end{array}$ & $\begin{array}{l}\text { Symptoms have improved significantly, but are not } \\
\text { completely alleviated. You may be able to tolerate some } \\
\text { fiber but might still have trouble digesting foods high } \\
\text { in fiber and fat. More fibrous foods are added in this } \\
\text { phase, in the form of soft cooked vegetables and pureed } \\
\text { beans/lentils. Use the foods list as a guide to help you } \\
\text { advance to this stage. Remember to drink plenty of } \\
\text { water and increase probiotic foods (e.g., plain yogurt, } \\
\text { aged cheese, fermented veggies, kefir, miso, microalgae, } \\
\text { pickles, honey, raw honey, fermented cabbage), when } \\
\text { adding fiber to your diet! }\end{array}$ & $\begin{array}{l}\text { Soft greens (butter lettuce, cooked collard greens, } \\
\text { baby spinach without stems), well-cooked } \\
\text { lean meats, aged cheeses (Cheddar, Gruyere, } \\
\text { Manchego, Gouda and Parmesan-types like } \\
\text { Parmigiano-Reggiano and Grana Padano), nut } \\
\text { butters, tomatoes, pureed berries with seeds } \\
\text { strained out, and foods baked with IBD-AID } \\
\text { friendly flours (bean flours, nut flours). }\end{array}$ \\
\hline $\begin{array}{l}\text { III. If in remission with no strictures, } \\
\text { can gradually go back to normal food } \\
\text { preparation. Can gradually increase } \\
\text { intact fiber intake. }\end{array}$ & $\begin{array}{l}\text { Your symptoms are gone. You are feeling stronger and } \\
\text { are becoming more comfortable eating a greater variety } \\
\text { of foods. Your bowel movements are well controlled } \\
\text { and solid. }\end{array}$ & $\begin{array}{l}\text { Stir-fried vegetables, cruciferous veg like } \\
\text { cabbage, cauliflower, and broccoli, meats, citrus } \\
\text { fruits, whole beans, and whole nuts. }\end{array}$ \\
\hline
\end{tabular}

IBD, inflammatory bowel disease; AID, anti-inflammatory diet. 
min E supplementation is summarized and presented in Ta-

ble 2 .
Olendzki et al. ${ }^{14}$ demonstrated a promising retrospective case series of AID as a treatment for IBD patients who did not

Table 2. Summary of Anti-Inflammatory Foods Studies

\begin{tabular}{|c|c|c|c|c|c|c|}
\hline Study (year) & Disease & $\begin{array}{l}\text { Anti-inflammatory diet/ } \\
\text { food }\end{array}$ & Study design & $\begin{array}{l}\text { No. of } \\
\text { IBD } \\
\text { patients }\end{array}$ & $\begin{array}{l}\text { Study } \\
\text { duration } \\
\text { (wk) }\end{array}$ & $\begin{array}{l}\text { Results on clinical } \\
\text { outcomes }\end{array}$ \\
\hline Ishikawa et al. $(2003)^{22}$ & UC & Fermented milk & Randomized clinical trial & 11 & 52 & $\begin{array}{l}\text { Maintenance of remission } \\
\text { and prevention of relapse }\end{array}$ \\
\hline Kato et al. $(2004)^{23}$ & UC & Fermented milk & $\begin{array}{l}\text { Randomized placebo } \\
\text { control trial }\end{array}$ & 20 & 12 & Induction of remission \\
\hline Lorea Baroja et al. $(2007)^{25}$ & $C D$ and $U C$ & Yogurt & Open-label study & 20 & 4 & $\begin{array}{l}\text { Induction and maintenance } \\
\text { of remission }\end{array}$ \\
\hline Shadnoush et al. $(2013)^{26}$ & $C D$ and $U C$ & Yogurt & Interventional study & 86 & 8 & Maintenance of remission \\
\hline Nyman et al. $(2020)^{29}$ & UC & Oat bran & $\begin{array}{l}\text { Randomized controlled } \\
\text { study }\end{array}$ & 47 & 24 & $\begin{array}{l}\text { No significant difference on } \\
\text { relapse rate }\end{array}$ \\
\hline Kanauchi et al. $(2003)^{32}$ & UC & $\begin{array}{l}\text { Germinated barley } \\
\text { foodstuff }\end{array}$ & Open trial, multicenter & 21 & 24 & $\begin{array}{l}\text { Decrease in clinical activity } \\
\text { index for long-term } \\
\text { administration }\end{array}$ \\
\hline Hanai et al. $(2004)^{33}$ & UC & $\begin{array}{l}\text { Germinated barley } \\
\text { foodstuff }\end{array}$ & $\begin{array}{l}\text { Open-label, non- } \\
\text { randomized trial }\end{array}$ & 59 & 52 & $\begin{array}{l}\text { Decrease in the cumulative } \\
\text { recurrence rate }\end{array}$ \\
\hline Faghfoori et al. $(2014)^{34}$ & UC & $\begin{array}{l}\text { Germinated barley } \\
\text { foodstuff }\end{array}$ & $\begin{array}{l}\text { Open-label, randomized } \\
\text { control trial }\end{array}$ & 46 & 8 & $\begin{array}{l}\text { Increased maintenance in } \\
\text { remission }\end{array}$ \\
\hline Brotherton et al. $(2014)^{35}$ & $C D$ & Wheat bran cereal & $\begin{array}{l}\text { Randomized controlled } \\
\text { single-blinded trial }\end{array}$ & 4 & 4 & $\begin{array}{l}\text { Increase in maintenance of } \\
\text { remission }\end{array}$ \\
\hline Grimstad et al. $(2011)^{38}$ & UC & Salmon fillet & Interventional pilot study & 12 & 8 & Induction of remission \\
\hline Schreiner et al. $(2019)^{46}$ & $\mathrm{IBD}$ & Gluten-free diet & $\begin{array}{l}\text { Prospective cross- } \\
\text { sectional study }\end{array}$ & 1,223 & - & $\begin{array}{l}\text { No significant difference on } \\
\text { disease activity }\end{array}$ \\
\hline Tasson et al. $(2017)^{51}$ & $C D$ & Meat & Cross-sectional study & 103 & 52 & Relapse of disease \\
\hline Barnes et al. $(2017)^{52}$ & UC & Fatty acids & $\begin{array}{l}\text { prospective, multicenter, } \\
\text { observational study }\end{array}$ & 412 & - & Increase disease flares \\
\hline Prince et al. $(2016)^{54}$ & $C D$ and $U C$ & Low FODMAP diet & Case note review study & 88 & 6 & Decreased disease severity \\
\hline Cox et al. $(2017)^{55}$ & $C D$ and $U C$ & Low FODMAP diet & $\begin{array}{l}\text { Randomized, double- } \\
\text { blind, placebo- } \\
\text { controlled, cross-over, } \\
\text { re-challenge trial }\end{array}$ & 32 & 3 day & $\begin{array}{l}\text { Worsened the } \\
\text { gastrointestinal } \\
\text { symptoms }\end{array}$ \\
\hline Saw et al. $(2019)^{57}$ & $\begin{array}{l}\text { DSS-induced } \\
\text { colitis mice }\end{array}$ & Vitamin E & Clinical trial & - & 20 day & Improved disease severity \\
\hline
\end{tabular}

IBD, inflammatory bowel disease; UC, ulcerative colitis; $C D$, Crohn's disease; FODMAP, fermentable oligosaccharide, disaccharide, monosaccharide, and polyol; DSS, dextran sulfate sodium. 
show any positive changes after pharmacological therapy and for patients where treatment (e.g., anti-inflammatory drugs or surgery) was not as effective. In the case series, 40 patients were offered AID while 11 patients completed AID therapy and contributed to the data required. All the patients experienced an improvement in their symptoms and downscaled their current medication therapy after adopting the diet for $>4$ weeks. ${ }^{14}$ However, there is a limitation concerning small sample size and study design, Thus, randomized clinical trials are needed to evaluate the efficacy of AID as adjunctive dietary therapy for IBD.

\section{Probiotics}

Probiotics are live microorganisms and when administered in adequate amounts, they can confer health benefits to the host. ${ }^{15}$ The effects of probiotics on the induction and maintenance of UC and CD have been investigated in several interventional trials, however, the results are inconsistent. Kruis et al. ${ }^{16}$ performed a double-blinded, double-dummy trial where UC patients were randomized to either receive probiotic capsules of Escherichia coli Nissle $200 \mathrm{mg}$ once daily $(\mathrm{n}=162)$ or mesalazine $500 \mathrm{mg}$ medication three times daily $(\mathrm{n}=165)$ for 12 months. At the end of the study, patients were assessed based on the histological, clinical and endoscopic activity indices. It was found that the efficacy and safety of $E$. coli Nissle were equivalent to the gold standard therapy (mesalazine) in maintaining the remission in UC patients. A meta-analysis conducted in 2014 which included 20 high-quality randomized control trials (RCTs) also showed probiotics could prevent relapse of UC as effectively as mesalazine (relative risk [RR], 1.0; 95\% confidence interval [CI], 0.79-1.26). ${ }^{17}$

The efficacy of probiotics was recommended for adjuvant use of multispecies probiotic VSL\#3 that contains 4 strains of Lactobacillus (L. casei, L. plantarum, L. acidophilus, and L. delbrueckii subsp. bulgaricus), 3 strains of Bifidobacterium (B. longum, B. breve, and B. infantis), and 1 strain of Streptococcus (S. salivarius subsp. thermophilus). For mild to moderately active UC patients, a meta-analysis conducted showed that VSL\#3, when added to conventional therapy at a daily dose of $3.6 \times 10^{12} \mathrm{CFU}$ (colony forming unit), could achieve clinical response and remission compared to the use of conventional therapy alone. ${ }^{18}$

In contrast, the Cochrane Collaboration review that included 12 studies ( $\mathrm{n}=1,473$ participants) showed that the effectiveness of probiotics in the management of UC patients remains uncertain. ${ }^{19}$ The review revealed no statistical differ- ence in the clinical relapse (RR, 1.01; 95\% CI, 0.84-1.22) and maintenance of remission (RR, 1.06; 95\% CI, 0.90-1.25) between probiotics and mesalazine. ${ }^{19}$ This could be due to the small sample size used for clinical trials that limit the number of data. Thus, no firm conclusion can be drawn on the efficacy of probiotics in maintaining remission of UC.

Among CD patients, 2 meta-analyses failed to demonstrate the efficacy of probiotics in the treatment of the disease..$^{20,21}$ Shen et al. ${ }^{20}$ reported that the supplementation with Lactobacillus $G G$ resulted in an increase in the relapse rate of $\mathrm{CD}$ compared with placebo (RR, 1.68; 95\% CI, 1.07-2.64). Similarly, Rahimi et al. ${ }^{21}$ reported on negative findings of probiotics efficacy in maintaining remission and preventing clinical and endoscopic relapse among $\mathrm{CD}$ patients.

To date, most of the studies investigated on the use of dietary supplements (capsules, tablets, and powders) compared with foods containing probiotics (yogurt, fermented and unfermented milk/dairy products, miso, tempeh) ${ }^{16-21}$ In this review, 4 studies that reported on the beneficial consumption of foods containing probiotics as a therapeutic strategy in the management of IBD were included. ${ }^{22-26}$

\section{1) Fermented Milk Supplementation}

Ishikawa et al. ${ }^{22}$ conducted a clinical trial to compare a group of UC patients provided with bifidobacteria supplemented fermented milk (BFM, $\mathrm{n}=11)$ and a control group $(\mathrm{n}=10)$ without supplementation of BFM, however, both groups received standard medications as a part of treatment. The BFM group ( $\mathrm{n}=11)$ was prescribed with $100 \mathrm{~mL} /$ day of commercially available BFM products containing B. breve, B. bifidum, and L. acidophilus for a 1-year study period. Colonoscopy, general blood marker test, and intestinal flora examination were performed at the beginning of the trial and the end of the study period. The exacerbation of symptoms was observed in 3 out of 11 subjects in the BFM group, and 9 out of 10 subjects in the control group. The statistical findings indicated a cumulative reduction of relapse rates in the BFM group $(P<0.05)$. However, the effectiveness of probiotic therapy among patients with active UC has not been investigated in a placebocontrolled study.

In 2004, Kato et al. ${ }^{23}$ conducted another randomized placebo-controlled trial among outpatients and hospitalized active UC patients from Nihon University School of Medicine, Tokyo, Japan. Significant improvement in both clinical and endoscopic activity indexes of mild to moderately active UC patients was noted. The trial was conducted among a group of 
UC patients with BFM supplementation $(\mathrm{n}=10)$ containing $B$. breve, B. bifidum, and Lactobacillus acidophilus, and a placebo group $(\mathrm{n}=10)$ for 12 weeks. ${ }^{23}$ The BFM group received 100 $\mathrm{mL} /$ day of BFM besides ongoing conventional treatment. It was concluded that BFM supplementation was safer and more effective compared with only conventional treatment in the management of active UC patients. ${ }^{23}$ However, this is the only pilot study available, hence more studies are required to confirm the results.

In a more recent study, a similar therapeutic strategy with a supplement that consisted of B. breve, B. bifidum, and L. acidophilus demonstrated a minimum effect of BFM in maintaining the remission of UC patients. ${ }^{24}$ A total of 195 patients with quiescent UC was randomized into the BFM group ( $\mathrm{n}=$ $98)$ and placebo group $(n=97)$. The BFM group was supplemented with $100 \mathrm{~mL} /$ day BFM for 48 days. At the end of the study, the relapse-free survival analysis conducted showed no significant difference between the BFM and placebo groups (hazard ratio, 1.16; 95\% CI, 0.63-2.14). No significant difference was also noted between the BFM group (22.7\%) and the placebo group $(20.0 \%)$ for the incidence of relapse with $P$-value of 0.651 .

\section{2) Yogurt}

Lorea Baroja et al. ${ }^{25}$ conducted an open-label study involving healthy $(n=20)$ and IBD $(n=20)$ subjects, with 15 and 5 of the IBD patients diagnosed with $\mathrm{CD}$ and UC, respectively. All the subjects were supplemented with yogurt containing probiotics namely $L$. reuteri and $L$. rhamnosus. After 30 days of treatment, the $\mathrm{CD}^{+} \mathrm{CD} 25^{\text {high }} \mathrm{T}$ cells proportion increased significantly. Meanwhile, the proportion of myeloid dendritic cells and tumor necrosis factor (TNF)- $\alpha$ /interleukin (IL)-12 monocytes content reduced in both UC and DC patients. ${ }^{25}$

Similarly, an interventional study conducted by Shadnoush et al. ${ }^{26}$ also revealed a positive effect of yogurt containing probiotic consumption on UC patients. Effect of consuming about $250 \mathrm{~g}$ of yogurt containing Bifidobacterium and Lactobacillus strains daily for 8 weeks were evaluated ${ }^{26}$ Stool samples were collected from the patients at the baseline and after the treatment. The changes in stool specimens were measured using Taqman real-time polymerase chain reaction method. The results showed an increase in the CFU means of Lactobacillus and Bifidobacterium in the treatment group compared with the placebo group. ${ }^{26}$

In summary, IBD patients should consider consuming supplements or foods containing probiotics in their daily diet for lower risk of IBD adverse effects. However, research on the effectiveness of probiotics as a treatment for IBD is limited and emerging. As there were many variations among available studies, further research is required to determine the most suitable probiotic strains, dosing regimens of probiotics and specific population of IBD patients who can benefit from probiotic supplementation. Probiotics should be considered as a part of a patient's healthcare besides medications, nutritional intervention, and lifestyle management.

\section{Prebiotics}

Prebiotics can be defined as selectively fermenting non-digestible food ingredients or substances that can specifically support the growth and activity of health-promoting bacteria that can colonize the gastrointestinal tract. ${ }^{27}$ With an advanced understanding of the interaction between diet, microbiome and host, Bindels et al. ${ }^{28}$ proposed a new definition of prebiotic. Prebiotic was defined as a non-digestible compound that can be metabolized by microorganisms in the gut which will modulate the composition and activity of gut microbiota, thus conferring a beneficial physiological effect on the host. However, the efficacy of prebiotics in IBD treatment is still unclear.

\section{1) Oat Bran}

Nyman et al. ${ }^{29}$ conducted a controlled multicenter study among a group of adult patients with quiescent UC. In this study, UC patients were randomized into an active group $(n=67)$ that received $60 \mathrm{~g}$ of oat bran equivalent to $20 \mathrm{~g}$ of dietary fiber/day and a control group $(n=63)$ that received a low-fiber wheatbased products that provided $5 \mathrm{~g}$ dietary fiber/day. ${ }^{29}$ After 24 week of study, the oat bran group had significantly higher fecal butyrate concentrations with gastrointestinal symptoms prevented. However, the relapse rate was similar for both groups.

In a previous study, the effect of indirect intake of $60 \mathrm{~g}$ of oat bran incorporated in bread which is equivalent to $20 \mathrm{~g}$ of dietary fiber/day by UC patients in remission $(n=22)$ was assessed. ${ }^{30}$ The main outcome of this oat bran intervention was reflected by short-chain fatty acids concentration in the stools Fifteen subjects had a $36 \%$ increase in the fecal butyrate concentration within 4 weeks of intervention and the butyrate concentration maintained throughout 12 weeks of the trial. During the trial, no patients showed signs of disease flare but significant improvement in IBD symptoms was noted as assessed by the Seo activity index. This study demonstrated that patients with quiescent UC should increase their fiber intake with the consumption of a diet rich in oat bran. However, fur- 
ther studies are required to determine the long-term benefits of using oat bran in maintenance therapy for UC patients.

\section{2) Germinated Barley Foodstuff}

Germinated barley foodstuff (GBF) is a type of prebiotic which is composed of mainly fibers (cellulose, hemicellulose, and lignin) and some proteins and lipids as dietary compounds. ${ }^{31}$ In 2003, Kanauchi et al. ${ }^{32}$ conducted a multicenter open trial to investigate a long-term administration effect of GBF on the treatment of active UC in addition to the standard treatment. A total of 21 patients with mild to moderate UC received 20-30 g of GBF for 24 weeks, resulting in a reduction of clinical activity of disease especially the degree of visible blood in stools and presence of nocturnal diarrhea compared with the control group $(P<0.05){ }^{32}$

The same group of researchers conducted another multicenter trial in 2004 to investigate the efficacy of GBF for maintenance therapy among UC patients. ${ }^{33}$ Patients were randomized into control group $(n=37)$ and GBF group $(n=22)$. For the treatment group, patients received $20 \mathrm{~g}$ /day of GBF with standard drug treatment, while the control group only received the standard drug therapy. Response to treatment was assessed by monitoring the clinical activity index and endoscopic score. In this trial, the recurrence rate was found to be lower in the GBF group compared to that of the control group with significantly higher clinical activity index at 3, 6, and 12 months of the trial were observed. The authors concluded that GBF is safe and effective to prolong the remission state in UC patients. ${ }^{33}$

In a more recent open-labeled RCT study conducted in 2014, 41 patients (GBF group: $\mathrm{n}=20$, control group: $\mathrm{n}=20$ ) with UC in the inactive state were involved. ${ }^{34}$ The GBF group patients received $30 \mathrm{~g}$ of GBF, three times daily in addition to conventional treatment for 2 months. A decrease in serum level of C-reactive protein along with reduced gastrointestinal symptoms such as abdominal pain and cramping were noted. ${ }^{34}$ Overall, intake of GBF in daily diet by UC patients showed improvements in clinical disease activity.

\section{3) Wheat Bran}

Brotherton et al..$^{35}$ conducted an interview-based single-armed study to evaluate the effect of wheat bran intake on CD patients health $(n=7)$. Patients were instructed to consume a half cup of whole wheat bran cereal daily, reduce the intake of refined carbohydrate and maintain sufficient fluid intake. At the end of the 4-week study, improvement in the clinical activ- ity scores as measured by the Harvey-Bradshaw index was reported. ${ }^{35}$ The authors concluded that consuming wheat bran in daily diet is feasible for CD patients and no adverse effects of the diet were reported.$^{35}$ However, the study has several limitations such as the use of small sample size and lack of objective biological measures such as fecal inflammatory markers, calprotectin, and/or lactoferrin.

In summary, the incorporation of dietary prebiotics in the treatment of IBD patients resulted in promising findings as reported in small-scale studies. As prebiotics are easy to administer, inexpensive, and lack of adverse effects, IBD patients should incorporate prebiotics $t$ as an adjunct to their medical treatment. However, more studies should be conducted to assess the effectiveness of prebiotics in a larger randomized controlled clinical studies for safe consumption of prebiotics by IBD patients as an alternative to standard therapeutics management of IBD. Furthermore, there is a need to further understand the mechanism of prebiotics and their interactions with the gut microbiota to identify specific prebiotics that is effective in the treatment of IBD.

\section{Omega-3 Fatty Acids}

Many studies have shown the beneficial effects of omega 3 fatty acids ( $\omega 3 \mathrm{FA})$ which includes eicosapentaenoic acid (EPA) and docosahexaenoic acid (DHA) as adjunctive in the treatment of IBD. However, there are many controversial effects of $\omega 3 \mathrm{FA}$ on $\mathrm{CD}$ or $\mathrm{UC}$, that were related to the amount of w3FA supplementation, variability in the sample size, and differences of methodologies in clinical trials. ${ }^{36}$ The consumption of $\omega 3 \mathrm{FA}$ was associated with anti-inflammatory properties that can improve mucosal healing of intestines and alleviate the active symptoms of IBD. ${ }^{37}$ The intake of essential $\omega 3$ FA can be achieved by regular consumption of foods such as oily fish (tuna, salmon, mackerel or sardine) or by supplementation with fish or olive oil.

An interventional pilot trial was conducted to assess the efficacy of high consumption of salmon fillet regularly ( $600 \mathrm{~g}$ per week) in mildly active UC patients $(\mathrm{n}=12)$ for 8 weeks. ${ }^{38}$ The efficacy of salmon fillet consumption was assessed using a Simple Clinical Colitis Activity Index (SCCAI), sigmoidoscopy, serum fecal calprotectin inflammatory markers, rectal biopsy, and plasma fatty acid (FA) profile evaluation before and after the dietary intervention. The findings revealed a reduction in the SCCAI and FA content in plasma and rectal specimen ${ }^{38}$ which indicates the beneficial effects of salmon fillet consumption on disease activity in patients with mild UC. ${ }^{38}$ 
In a different study by Scaioli et al., ${ }^{39}$ a placebo-controlled trial involving UC patients in remission was conducted to evaluate the efficacy of EPA-free FA capsules supplement (EPA-FFA) to reduce gut inflammation. In the study, patients were randomly assigned into 2 groups which were (1) patients consuming capsules supplement with EPA-FFA, 500 $m g$, twice per day $(n=30)$, and (2) placebo $(n=30)$ for 6 months of the study period. Assessment of fecal calprotectin and clinical parameters was performed at baseline of the study, after 3 months, and after 6 months. ${ }^{39}$ Administration capsules supplement of EPA-FFA for 6 months was found to reduce calprotectin in fecal without adverse effects. EPA-FFA was found to be beneficial in inducing and maintaining symptom-free remission in patients with UC. However, further investigations involving a larger sample size are needed.

In another study, an open-label clinical trial was conducted to investigate the safety and efficacy of $\omega 3$ emulsified formulation when consumed by $\mathrm{CD}$ patients in remission $(\mathrm{n}=5){ }^{40} \mathrm{~Pa}-$ tients were required to ingest one bottle $(100 \mathrm{~mL})$ of commercially available $\omega 3$ emulsifying test formulation daily for 28 days. The results showed that supplementation with the test formulation is safe with minimal side effects. CD activity index scores decreased after ingestion of one bottle of the test formulation (containing $600 \mathrm{mg}$ of EPA and $260 \mathrm{mg}$ of DHA) and blood tests revealed no serious adverse effects. ${ }^{40}$ However, this study involved a small sample size which is insufficient to completely elucidate the effects of the $\omega 3$ emulsifying formulation to maintain the remission state. ${ }^{40}$

Although some clinical trials have demonstrated the efficacy of $\omega 3$ FA supplementation in the treatment of IBD, a review by Lev-Tzion et al. ${ }^{41}$ showed controversial effects of $\omega 3$ FA. LevTzion et al. ${ }^{41}$ systematically reviewed the safety and efficacy of $\omega 3 \mathrm{FA}$ for maintaining remission in CD. Six studies were included in the analysis with a total of 1,039 patients and the primary study outcome was relapse rate. Based on 2 large highquality studies included in the review, $\omega 3 \mathrm{FA}$ was probably found ineffective for the maintenance of remission in CD.

Controversial findings on the effects of $\omega 3 \mathrm{FA}$ as adjunctive in the treatment of CD and UC could be due to the small number of patients, mode of supplementation consumption and types of $\omega 3 \mathrm{FA}$ formulation used. Therefore, further research is warranted to elucidate the influence of $\omega 3 \mathrm{FA}$ supplementation on the clinical course of the disease and to establish daily recommended intake of $\omega 3 \mathrm{FA}$ for desirable induction of remission and prevention of relapse in IBD.

\section{Restriction of Refined Carbohydrates and Gluten- Based Product Intake}

Dietary surveys reported that about $10 \%$ of IBD was associated with sugary foods or refined carbohydrates which aggravated IBD symptoms and trigger the relapse of the disease. ${ }^{42,43}$ However, there is limited control data to support the impact of exclusions diets of refined carbohydrates on IBD. In the present review, few interventional studies that investigated the impact of refined carbohydrates in comparison to control diet for maintenance of remission in inactive $\mathrm{CD}$ patients were summarized. ${ }^{44,45}$

Jones et al. ${ }^{44}$ conducted a controlled trial involving inactive CD patients $(n=20)$ for a month trial. The study showed that none out of $10 \mathrm{CD}$ patients on unrefined carbohydrate fiberrich diet (control diet) remained in the remission state compared with 7 out of 10 patients on the exclusion diet. ${ }^{44}$ However, the study failed to explain the methods used for random allocation concealment which could influence the study findings.

Another controlled trial was conducted by Ritchie et al. ${ }^{45}$ to assess the effects of 2 different diets namely refined carbohydrates and unrefined carbohydrates on CD patients $(n=352)$. Patients were randomized to consume either carbohydrate in the refined form, e.g. rice and white flour $(\mathrm{n}=162)$ or carbohydrates in the natural unrefined state $(\mathrm{n}=190)$. Over the 2-year trial, no clear differences were seen in the clinical course of patients in both groups as assessed by gastrointestinal symptoms improvement, stool count, and weight changes. ${ }^{45} \mathrm{How}$ ever, there was a systematic error due to attrition bias as patients from the intervention group withdrew from the study by 4 -fold compared to the control group due to non-compliance.

Besides refined carbohydrates, gluten intake is also restricted for IBD patients. Gluten is a protein mainly found in processed wheat and related grain species (rye and barley). Wheat products such as bread, cakes, and pastries commonly contain gluten. A gluten-free diet (GFD) was associated with improved symptoms in IBD patients, however, controlled trials are limited to conclude the usefulness of this restrictive diet. Schreiner et al. ${ }^{46}$ conducted a cross-sectional analysis involving IBD patients $(n=1,254)$ where GFD was adopted by approximately $5 \%$ of patients, with most of them not being diagnosed with celiac disease.

Despite patients' belief in a favorable clinical course of the disease, Schreiner et al. could not objectify the impact of GFD on the disease course. Herfarth et al. ${ }^{47}$ demonstrated that $65.6 \%$ of IBD patients who attempted GFD described improvements in gastrointestinal symptoms and $38.3 \%$ reported 
with fewer or less severe flares of IBD. These 2 cross-sectional analyses could not be used to conclude long-term effects of GFD on the course of the disease.

\section{Restriction of Lactose-Based Food Intake}

Lactose is a type of sugar naturally found in milk and dairy products that are hydrolyzed by lactase to be readily absorbed in the intestine. Most of the studies that described the restriction of lactose-based foods adopted by IBD patients are based on cross-sectional analysis. Findings by Cohen et al. ${ }^{48}$ as a result of an interview with CD patients $(n=105)$ indicated significant avoidance of milk consumption which was associated with their beliefs that milk worsens their symptoms. In the study, only 6 CD patients reported that the consumption of milk does affect their symptoms.

In a study conducted in New Zealand to evaluate the effect of dairy food intake in IBD patients $(n=165)$, Nolan-Clark et al. ${ }^{49}$ reported that most the CD patients (67.3\%) stated that the consumption of dairy products had no adverse effect on selfreported CD symptoms. Nevertheless, CD patients who experienced an adverse reaction after consuming products containing milk reported that dairy products with high-fat content (cream, ice-cream, and cheese) worsen the perceived CD symptoms.

\section{Reduction of High-Fat Food Intake}

Dietary intake of high-fat foods mainly saturated fat (e.g., fatty beef, lamb, pork, poultry with skin, deep-fried foods) and $\omega 6 \mathrm{FA}$ rich foods (e.g., red meat, margarine, cooking oil) can induce intestinal inflammation and growth of pathogenic bacteria that can further stimulate the immune system towards a proinflammatory state.$^{50}$ IBD patients often modify their diets by frequently avoiding fatty foods during the remission to prevent the flares of the disease. ${ }^{10}$

In a cross-sectional study, Tasson et al. ${ }^{51}$ analyzed the effect of dietary intake among CD and UC patients in remission $(\mathrm{n}=53)$ and active disease $(\mathrm{n}=50)$ states using 146-food items self-administered food frequency questionnaire over a 1 year of the study period. The outcomes of the clinical state of disease were assessed using fecal calprotectin. A positive association between the risk of relapse and consumption of meat (OR, 3.61; 95\% CI, 1.15-11.38) was found which indicated the detrimental effects of meat on disease relapse.

In another study, Barnes et al. ${ }^{52}$ conducted a prospective observational study among UC patients in remission $(n=412)$ who were on aminosalicylates treatment to investigate the re- lationship between high dietary intake of specific fatty acids with the risk of disease flares. The dietary intake was assessed using a validated food frequency questionnaire at the baseline and follow-up was performed for a year. The disease activity of UC was assessed using the SCCAI. The study demonstrated that $11 \%(n=45)$ of UC patients experienced a flare of disease within the 12-month study period. The output of multivariable logistic regression analysis indicated that the higher the intake of myristic acid (saturated fat commonly found in coconut oil, palm oil, and dairy fats), the higher the odds of relapse (OR, $3.01 ; 95 \%$ CI, $1.17-7.74){ }^{52}$

\section{Low FODMAPs Diet}

The FODMAP diet stands for fermentable oligosaccharide, disaccharide, monosaccharide, and polyol. FODMAP exclusion diet in IBD patients was based on the notion that high fermentable carbohydrates (e.g., glucose, fructose, sucrose, lactose, or polyols) are poorly absorbed in the intestinal tract, thus they are rapidly fermented by gut bacteria giving rise to a high osmotic load. ${ }^{53,54}$

Prince et al. ${ }^{54}$ conducted a prospective case-note review to determine the efficacy of a low FODMAP diet in the management of functional-like gastrointestinal symptoms (FGS) among IBD patients. In the study, IBD patients $(\mathrm{n}=88)$ were advised to follow a diet low in FODMAP for a minimum of 6 weeks by a specialist gastrointestinal dietitian. About $78 \%$ of patients reported improvement of their symptoms during follow-up sessions as assessed by the Gastrointestinal Symptoms Rating Scale. Besides, a significant reduction $(P<0.001)$ in the severity of abdominal pain, heartburn, flatulence, bloating, bleaching, incomplete evacuation, and nausea was observed. Not only that, improvements in stool consistency $(P=0.002)$ and frequency $(P<0.001)$ was also assessed using the Bristol Stool Form Scale. ${ }^{54}$

In a different study, Cox et al..$^{55}$ used a randomized, doubleblinded, placebo-controlled and re-challenge trial to evaluate the effect of individual fermentable carbohydrates on the FGS among IBD patients in remission $(\mathrm{n}=32)$ who were experiencing co-existence of FGS. The study reintroduced fructans (12 g/day), galactooligosaccharides (6 g/day) and sorbitol (6 g/day) over a 3-day re-challenge period and glucose placebo ( $12 \mathrm{~g} /$ day) while monitoring the gastrointestinal symptoms. The RCT findings showed that the intake of fructans exacerbated the gastrointestinal symptoms of IBD patients compared to placebo, galactooligosaccharides and sorbitol. ${ }^{55}$ 


\section{Vitamin E Rich Foods Supplementation}

Vitamin E is a group of phytochemicals which includes tocopherols (TPs), tocotrienols (T3s), and their derivatives are naturally derived from plant-based sources such as nuts, seeds, green leafy vegetables and plant oils. ${ }^{56}$ Vitamin E, a lipid-soluble antioxidant compound in a cellular membrane is recognized to protect membrane lipids from lipid peroxidation by scavenging the chain-carrying lipid peroxyl radicals57 and have prominent anti-inflammatory effects. ${ }^{57}$

In a study where dextran sulfate sodium (DSS)-induced UC mice were used, the severity of IBD disease improved with the supplementation of an oral tocotrienol-rich fraction (TRF) as characterized by the reduction in the disease activity index and histopathological inflammatory scoring. ${ }^{57}$ Supplementation of TRF (containing $70 \%$ of $\alpha \mathrm{T} 3$, a dose of $150 \mathrm{mg} / \mathrm{kg} / \mathrm{day}$ ) was compared against the $\alpha$-tocopherol ( $\alpha \mathrm{TP}$; a dose of 150 $\mathrm{mg} / \mathrm{kg} /$ day) for 20 days. TRF demonstrated anti-inflammatory and antioxidant properties as indicated by the reduction of inflammation and oxidative biomarkers including IL-6, IL-17, TNF- $\alpha$, myeloperoxidase (MPO), cyclooxygenase-2 (COX-2), nitrite oxide (NO), malondialdehyde (MDA), and pNF-Kb. Meanwhile, for treatment with aTP, the MPO and inflammatory markers, no changes were observed. ${ }^{57}$ The authors proposed clinical study to be conducted to evaluate the effectiveness of TRF among UC patients.

\section{WHAT CLINICIANS SHOULD ADVISE THEIR PATIENTS ON THE AID?}

Table 3 summarizes the general recommended diet for IBD patients including the specific recommendations for $\mathrm{CD}$ and UC patients. However, there is limited information to guide clinicians on the use of specific dietary therapy as an adjunctive treatment for $\mathrm{CD}$ and UC.

Table 3. General Summary of Recommended Diet for CD and UC Patients

General aspects of the diet for IBD

1. Diet is highly individualized.

2. Eat smaller meals of high energy and nutrient density, consumed at frequent intervals.

3. Stay hydrated, drink a small amount of water throughout the day.

4. Maximize the energy and protein intake for maintenance of weight and replenishment of nutrients while tailoring to the patient's current bowel function.

5. Encouraged to follow a normal diet as possible which is high quality and balanced whenever possible.

6. When the appetite is poor, food fortification or nutritional supplement use may be necessary.

7. During disease flares, the patient may need to eliminate foods that cause symptoms such as lactose-containing foods, high fiber foods, and highfat foods.

8. If lactose is avoided, encourage alternate high calcium and vitamin D food sources (tofu, collard greens, low lactose cheese or lactose-free milk, fortified milk substitutes).

9. During a disease flare when the intake of low insoluble fiber is beneficial, consider the intake of fruits and vegetables that are easier to digest (e.g., soft, cooked, avoid the skins and seeds).

10. Avoid unnecessary dietary restrictions.

Dietary management of $C D$

1. For active $C D$, withdraw the normal diet for 2-4 weeks and replace it with liquid formula diet (elemental, peptide, or polymeric diets), or parenteral nutrition can be used to induce remission in some of the patients.

2. For maintenance of remission state in $C D$, following the period of normal food withdrawer, the transition of normal foods need to be made with care; foods should be reintroduced gradually for 2-4 weeks with the gradual cessation of the formula diet.

3. For CD patients with stricturing, a low insoluble fiber diet (e.g., less raw fruits and vegetables, wholegrain) may be helpful to lessen the symptoms such as abdominal pain and diarrhea.

Dietary management of UC

1. For active UC, patients should be encouraged to reduce the foods that may increase the symptoms (e.g., insoluble fiber, concentrated sweets, high fat, caffeine/alcohol, and sugar alcohol). Also, patients are encouraged to increase their fluid intake.

2. For the remission state of UC, patients should be encouraged to consume varied, well-balanced diet and avoid unnecessary food exclusion.

CD, Crohn's disease; UC, ulcerative colitis; IBD, inflammatory bowel disease. 
Patient's perceptions of the benefits and harms of selected foods can impact their choice of food intake which can further influence their nutritional status. A majority of IBD patients $(\sim 60 \%)$ perceived diet/food as the risk factor for the relapse of IBD. ${ }^{8,58}$ Specifically, foods such as milk/dairy, fried foods/fatty foods, spicy foods, vegetables, nuts, red meats, high fiber foods, and coffee were frequently reported to worsen the IBD symptoms. ${ }^{48}$ IBD patients should focus on dietary pattern modifications compared to exclusion of specific foods particularly the nutrient-dense foods.

As the IBD-AID requires the elimination of specific foods or food groups, it may inadequately replete the nutrients deficiencies especially for those IBD patients who are already at the risk of malnutrition. Patients and clinicians should have reservations about the practicality of AID to maintain the dietary therapy over a long period as AID could lead to financial burden or reduction in overall calorie intake.

\section{Potential Nutritional Inadequacy of AID}

During dietary consultation, clinicians should carefully discuss with patients regarding insufficient nutrient intake. Macro- and micro-nutrient deficiencies can occur as a result of excessive self-imposed dietary restrictions of AID. First, IBD patients who strictly follow long-term restriction of processed complex carbohydrates may be at risk of inadequate calorie intake and weight loss. ${ }^{59}$

Secondly, excluding gluten-based products is one of the rec- ommendations for AID. Nevertheless, it is not commonly advised as IBD patients may practice unnecessary exclusion and perhaps confuse symptoms with food allergies. ${ }^{47}$ Gluten-based cereals such as wholegrain-wheat based bread and brown rice are the major source of dietary fiber, thus IBD patients who practice GFD may be at risk of consuming inadequate fiber intake. ${ }^{60}$ Taetzsch et al. ${ }^{61}$ also found that healthy dietary recommendation developed with gluten-free menus have significantly lower protein, magnesium, potassium, vitamin E, folate, and sodium contents compared to gluten-containing menus. Furthermore, recent surveys conducted on the nutritional quality of gluten-free products available in markets also showed low content of protein with high fat and salt content compared to their equivalent gluten-containing products. ${ }^{62}$

Thirdly, the restriction of lactose-based products as part of AID has potential for the insufficient intake of calcium and vitamin D. Vernia et al. ${ }^{63}$ analyzed the means of the 22 -item quantitative questionnaire of calcium intake in 187 IBD patients and confirmed that the restriction of dairy products contributed to a lower intake of calcium in IBD patients compared to healthy controls. The main concern related to calcium and vitamin D deficiency is the adverse effect on bone health. Restriction of the dairy product might impact negatively on bone mineral density which in turn can increase the risk of osteoporosis. ${ }^{64}$ The restriction of dairy-based products also contribute to inadequate intake of protein, potassium, vitamin A, vitamin $B_{12}$, phosphorus, zinc and riboflavin. ${ }^{65}$

Table 4. What Should Clinicians Inform IBD Patients?

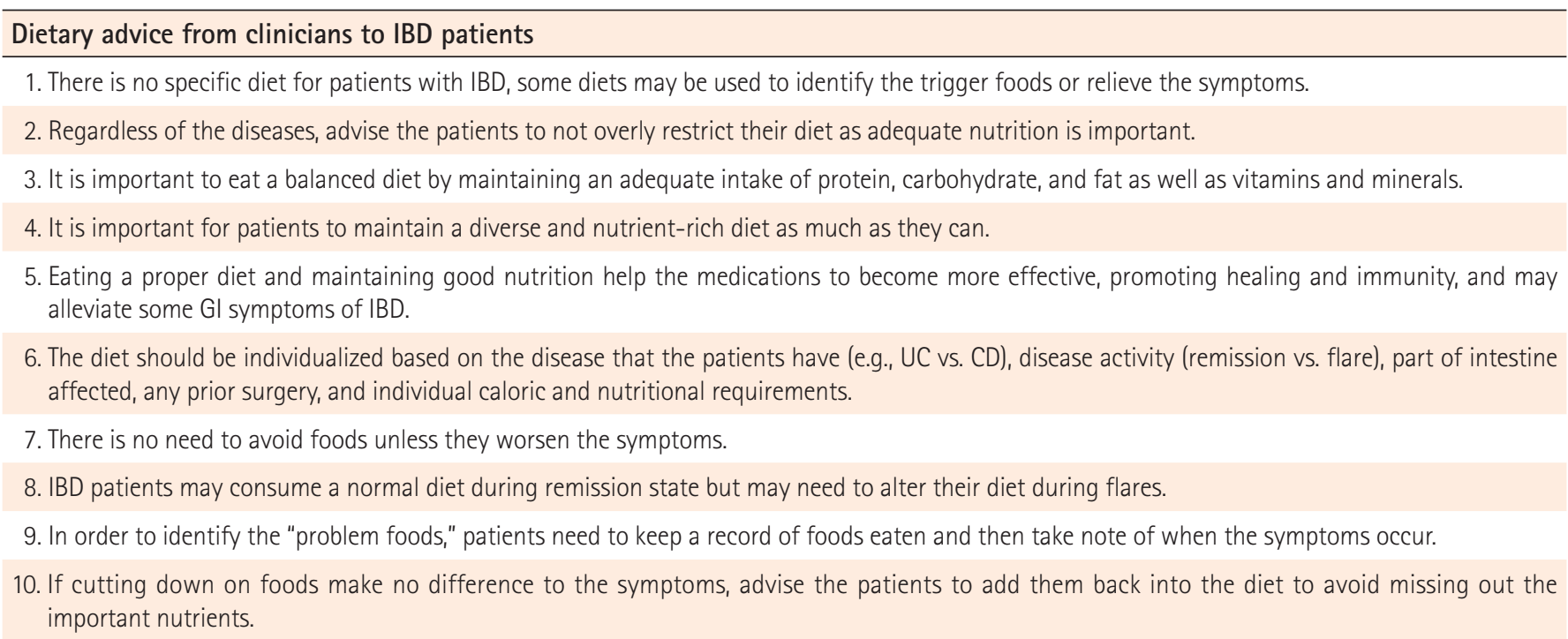

IBD, inflammatory bowel disease; GI, gastrointestinal; UC, ulcerative colitis; $C D$, Crohn's disease. 


\section{Recommendations for IBD Patients}

IBD patients frequently request for recommendations about food and diet besides disease improvement strategies during consultations with their clinicians. The most encountered question is "what to eat" which is the most difficult question to answer by many clinicians. In the present review, a general dietary guideline for IBD patients is presented in Table 4 . For clinicians, when advising patients on AID, it will be very helpful

Table 5. What IBD Patients Should Eat?

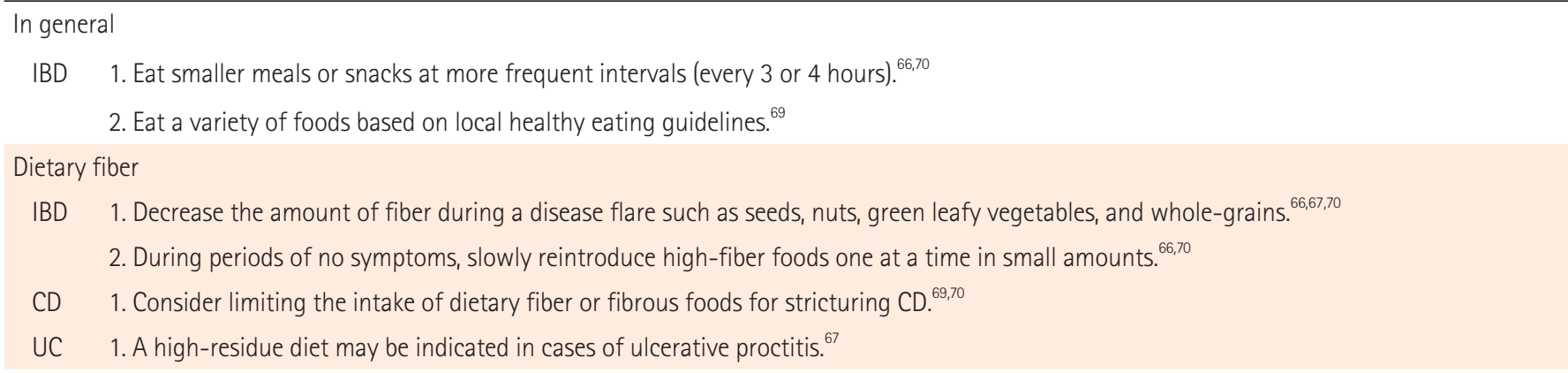

Vegetables and fruits

IBD 1. Avoid vegetables that are gas-producing when the disease is active such as broccoli, cauliflower, cabbage. ${ }^{70}$

2. When experiencing a disease flare, cooked, pureed, and peeled fruits and vegetables are better-tolerated. ${ }^{66,70}$

Milk and dairy-based foods

IBD 1. Maintain dairy product intake unless the intolerance develops. ${ }^{67}$

2. Limit or eliminate the intake of milk and milk products if patients do not digest dairy foods well, or are lactose intolerant. ${ }^{6,70}$

High-fat foods

IBD 1. Limit fats and oils to less than 8 teaspoons per day. ${ }^{66}$

2. If patients have diarrhea or bloating, reduce the fatty, greasy or fried foods. ${ }^{70}$

Carbohydrates

IBD 1. Reducing dietary fermentable oligosaccharides, disaccharides, and monosaccharides and polyols (FODMAPs) may reduce symptoms of IBD. ${ }^{67,70}$

Meat or protein-based foods

IBD 1. Choose the lean source of protein such as fish, chicken, eggs, or tofu as it is may be more tolerable. ${ }^{70}$

2. Avoid fatty, greasy or highly processed meats such as sausages, nuggets. ${ }^{66,70}$

3. Tender, well-cooked meat prepared without added fat. ${ }^{66}$

Beverages

IBD 1. Drink enough fluids (at least 8 cups each day) to avoid dehydration. ${ }^{66,70}$

2. Avoid high sugary drinks, sweet juices, caffeine, and alcohol when the disease is active. ${ }^{66,70}$

Probiotics

IBD 1. Eat foods with added probiotics and prebiotics. ${ }^{66}$

CD 1. Probiotics should not be used in the treatment of active disease. ${ }^{68,69}$

UC 1. Probiotic therapy can be considered for patients with mild to moderate UC. $^{68}$

Dietary supplements

IBD 1. Iron supplementation is recommended in all IBD patients when iron deficiency anemia is present. ${ }^{68,69}$

2. Calcium and vitamin $D$ supplementation are encouraged for those on steroid therapy. ${ }^{69,70}$

3. Folic acid supplementation for those treated with sulphasalazine. ${ }^{68}$

$\mathrm{CD} \quad$ 1. Vitamin $B_{12}$ supplementation with more than $20 \mathrm{~cm}$ distal ileum resection. ${ }^{68}$

IBD, inflammatory bowel disease; $C D$, Crohn's disease; UC, ulcerative colitis.

Adapted from: clinical practice guidelines ${ }^{66-69}$ and informal dietary suggestion. ${ }^{70}$ American Dietetic Association (ADA) ${ }_{i}^{66}$ World Gastroenterology Organization (WGO) ${ }^{67}$ European Society for Clinical Nutrition and Metabolism (ESPEN): ${ }^{68}$ British Dietetic Association (BDA) ${ }^{69}$ Crohn's and Colitis Foundation of America $(\mathrm{CCFA}){ }^{70}$ 
to consider several factors including the severity of patient's current clinical conditions, assessment of nutritional status before AID implementation, patient's willingness to adhere with AID for long-term, and also the feasibility of the AID itself (e.g., cost of the specialty foods, food preparations constraints, and challenges during social gathering). As there is no specific diet designed to treat IBD, general diet for IBD patients as proposed by medical societies in the form of clinical practice guidelines related to IBD were summarized and presented in Table $5{ }^{66-70}$

The elimination of specific foods from IBD-AID is often manageable by patients but adding unfamiliar foods particularly foods containing probiotics can be a huge barrier in maintaining compliance which might be due to cultural diversity of food choice. Clinicians also should be aware that all the anti-inflammatory foods reviewed here do not apply for all IBD patients. The intake of a particular food according to IBDAID can worsen clinical outcomes of some patients but may be beneficial for other patients. Therefore, personalized diets might be more effective in managing IBD and must be tailored based on current therapy and the natural history of the disease. Clinicians may instruct patients to be more vigilant of their food intake and take note of any associated symptoms by recording in food diaries. Personalized dietary modifications can identify specific triggers related to symptoms and also empower patients with a sense of control over their symptoms.

IBD patients who intend to implement AID first should be counselled on adequate calorie intake and emphasized on macro-and micro-nutrients intake such as protein, iron, calcium, vitamins $\mathrm{D}, \mathrm{B}_{12}$ and $\mathrm{A}$, folic acid, and zinc. Without proper medical/dietitian's advice, following the IBD-AID can predispose patients into nutritional deficiencies. Dietary education should emphasize on the importance of nutritional adequacy for a long-term.

Besides, alternative sources of dairy products with low lactose content (e.g., fermented lactose-free yogurt and milk, almond, rice or soy milk, and low-lactose cheese) can be suggested by clinicians. Even if someone with IBD is also intolerant to lactose, it is possible to safely consume certain dairy products that contain minimum lactose content and a good source of dietary calcium. Besides, the inclusion of other food sources of calcium such as salmon, sardine, broccoli, green leafy vegetables, tofu, and nuts can also help to boost the intake of calcium. Furthermore, incorporating calcium-fortified products in the diet of IBD patients can result in a sub-optimal intake of other key nutrients such as protein, magnesium, potassium, riboflavin and vitamin $B_{12}$.

Meanwhile, the restriction of gluten products should be accompanied by the consumption of well-balanced and nutrient-dense foods. Clinicians should encourage patients to consume various green leafy vegetables, asparagus, broccoli, cauliflower, lentils, meats, fish, and shellfish to obtain an adequate intake of folic acid and iron. Also, incorporation of fruits, vegetables, and nuts can increase the intake of dietary fiber. Furthermore, clinicians can guide their patients by recommending consumption of fortified or enriched gluten-free foods that contain sub-optimal levels of vitamins and minerals. Dietary supplements including iron, vitamin $\mathrm{B}_{12}$ and folate can be suggested for IBD patients to prevent nutritional deficiency.

Not only that, it is very important for close monitoring during the implementation of AID. Regular follow-up is advised throughout the phase of food elimination and during the phase of dietary modification. This is to individualize the nutritional therapy of AID based on disease activity, comorbidities, and complications-related IBD. Clinicians should be able to engage their patients in discussions to educate them on AID.

\section{CONCLUSIONS}

This review highlights the implications of AID on clinical outcomes of IBD patients. Lack of consistent data to support a practical recommendation of AID in managing IBD patients was noted due to various limitations present in previous research. It is necessary for clinicians advising IBD patients to have in-depth knowledge about the strengths and limitations of the IBD-AID nutritional regime to ensure its effectiveness as a therapeutic strategy. More prospective controlled clinical trials are required to provide accurate dietary recommendations to patients. This review is expected to assist clinicians to have a better understanding of an AID as an adjunct to the limited existing dietary recommendation for IBD.

\section{ADDITIONAL INFORMATION}

\section{Funding Source}

The authors received no financial support for the research, authorship, and/or publication of this article.

\section{Conflict of Interest}

Raja Ali RA is an editorial board member of the journal but 
did not involve in the peer reviewer selection, evaluation, or decision process of this article. No other potential conflicts of interest relevant to this article were reported.

\section{Author Contribution}

Conceptualization, methodology, writing - original draft preparation: Shafiee NH. Writing-review and editing: Shafiee NH, Manaf ZA, Mokhtar NM, Raja Ali RA. Approval of final manuscripts: all authors.

\section{Non-Author Contribution}

We are grateful to Barbara Olendzki, RD MPH, Director of the Center for Applied Nutrition UMass Medical School for her willingness to share the modified version of IBD-AID phases to be included in this review (Table 1).

\section{ORCID}

Shafiee NH

https://orcid.org/0000-0002-5233-1384

Manaf ZA

Mokhtar NM

Raja Ali RA https://orcid.org/0000-0002-5657-8608 https://orcid.org/0000-0002-4863-100X https://orcid.org/0000-0003-4995-3868

\section{REFERENCES}

1. Baumgart DC, Sandborn WJ. Inflammatory bowel disease: clinical aspects and established and evolving therapies. Lancet 2007;369:1641-1657.

2. Mulder DJ, Noble AJ, Justinich CJ, Duffin JM. A tale of two diseases: the history of inflammatory bowel disease. J Crohns Colitis 2014;8:341-348.

3. Lichtenstein GR, Loftus EV, Isaacs KL, Regueiro MD, Gerson LB, Sands BE. ACG clinical guideline: management of Crohn's disease in adults. Am J Gastroenterol 2018;113:481-517.

4. Rubin DT, Dubinsky MC, Martino S, Hewett KA, Panés J. Communication between physicians and patients with ulcerative colitis: reflections and insights from a qualitative study of in-office patient-physician visits. Inflamm Bowel Dis 2017; 23:494-501.

5. Wilhelm SM, Love BL. Management of patients with inflammatory bowel disease: current and future treatments. Clin Pharm 2017;9(3). https://doi.org/10.1211/CP.2017.20202316.

6. Neuman MG, Nanau RM. Inflammatory bowel disease: role of diet, microbiota, life style. Transl Res 2012;160:29-44.

7. David LA, Maurice CF, Carmody RN, et al. Diet rapidly and reproducibly alters the human gut microbiome. Nature 2014;505:559563.
8. Triantafillidis JK, Vagianos C, Papalois AE. The role of enteral nutrition in patients with inflammatory bowel disease: current aspects. Biomed Res Int 2015;2015:197167.

9. Limdi JK. Dietary practices and inflammatory bowel disease. Indian J Gastroenterol 2018;37:284-292.

10. Green N, Miller T, Suskind D, Lee D. A review of dietary therapy for IBD and a vision for the future. Nutrients 2019;11:947.

11. Pigneur B, Ruemmele FM. Nutritional interventions for the treatment of IBD: current evidence and controversies. Therap Adv Gastroenterol 2019;12:1756284819890534.

12. Gophna U, Sommerfeld K, Gophna S, Doolittle WF, Veldhuyzen van Zanten SJ. Differences between tissue-associated intestinal microfloras of patients with Crohn's disease and ulcerative colitis. J Clin Microbiol 2006;44:4136-4141.

13. Ott SJ, Musfeldt M, Wenderoth DF, et al. Reduction in diversity of the colonic mucosa associated bacterial microflora in patients with active inflammatory bowel disease. Gut 2004;53: 685-693.

14. Olendzki BC, Silverstein TD, Persuitte GM, Ma Y, Baldwin KR, Cave D. An fermentable oligosaccharide, disaccharide, monosaccharide, and polyol as treatment for inflammatory bowel disease: a case series report. Nutr J 2014;13:5.

15. Hill C, Guarner F, Reid G, et al. Expert consensus document. The International Scientific Association for Probiotics and Prebiotics consensus statement on the scope and appropriate use of the term probiotic. Nat Rev Gastroenterol Hepatol 2014;11:506-514.

16. Kruis W, Fric P, Pokrotnieks J, et al. Maintaining remission of ulcerative colitis with the probiotic Escherichia coli Nissle 1917 is as effective as with standard mesalazine. Gut 2004;53: 1617-1623.

17. Fujiya M, Ueno N, Kohgo Y. Probiotic treatments for induction and maintenance of remission in inflammatory bowel diseases: a meta-analysis of randomized controlled trials. Clin J Gastroenterol 2014;7:1-13.

18. Mardini HE, Grigorian AY. Probiotic mix VSL\#3 is effective adjunctive therapy for mild to moderately active ulcerative colitis: a meta-analysis. Inflamm Bowel Dis 2014;20:1562-1567.

19. Iheozor-Ejiofor Z, Kaur L, Gordon M, Baines PA, Sinopoulou V, Akobeng AK. Probiotics for maintenance of remission in ulcerative colitis. Cochrane Database Syst Rev 2020;3:CD007443.

20. Shen J, Ran HZ, Yin MH, Zhou TX, Xiao DS. Meta-analysis: the effect and adverse events of Lactobacilli versus placebo in maintenance therapy for Crohn disease. Intern Med J 2009; 39:103-109.

21. Rahimi R, Nikfar S, Rahimi F, et al. A meta-analysis on the effi- 
cacy of probiotics for maintenance of remission and prevention of clinical and endoscopic relapse in Crohn's disease. Dig Dis Sci 2008;53:2524-2531.

22. Ishikawa H, Akedo I, Umesaki Y, Tanaka R, Imaoka A, Otani T. Randomized controlled trial of the effect of bifidobacteria-fermented milk on ulcerative colitis. J Am Coll Nutr 2003;22:5663.

23. Kato K, Mizuno S, Umesaki Y, et al. Randomized placebocontrolled trial assessing the effect of bifidobacteria-fermented milk on active ulcerative colitis. Aliment Pharmacol Ther 2004;20:1133-1141.

24. Matsuoka K, Uemura Y, Kanai T, et al. Efficacy of bifidobacterium breve fermented milk in maintaining remission of ulcerative colitis. Dig Dis Sci 2018;63:1910-1919.

25. Lorea Baroja M, Kirjavainen PV, Hekmat S, Reid G. Anti-inflammatory effects of probiotic yogurt in inflammatory bowel disease patients. Clin Exp Immunol 2007;149:470-479.

26. Shadnoush M, Shaker Hosseini R, Mehrabi Y, et al. Probiotic yogurt affects pro- and anti-inflammatory factors in patients with inflammatory bowel disease. Iran J Pharm Res 2013;12: 929-936.

27. Gibson GR, Willems A, Reading S, Collins MD. Fermentation of non-digestible oligosaccharides by human colonic bacteria. Proc Nutr Soc 1996;55:899-912.

28. Bindels LB, Walter J, Ramer-Tait AE. Resistant starches for the management of metabolic diseases. Curr Opin Clin Nutr Metab Care 2015;18:559-565.

29. Nyman M, Nguyen TD, Wikman O, Hjortswang H, Hallert C. Oat bran increased fecal butyrate and prevented gastrointestinal symptoms in patients with quiescent ulcerative colitis: a randomized controlled trial. Crohns Colitis 360 2020;2:otaa005.

30. Hallert C, Björck I, Nyman M, Pousette A, Grännö C, Svensson H. Increasing fecal butyrate in ulcerative colitis patients by diet: controlled pilot study. Inflamm Bowel Dis 2003;9:116121.

31. Rasmussen HE, Hamaker BR. Prebiotics and inflammatory bowel disease. Gastroenterol Clin North Am 2017;46:783-795.

32. Kanauchi O, Mitsuyama K, Homma T, et al. Treatment of ulcerative colitis patients by long-term administration of germinated barley foodstuff: multi-center open trial. Int J Mol Med 2003;12:701-704.

33. Hanai H, Kanauchi O, Mitsuyama K, et al. Germinated barley foodstuff prolongs remission in patients with ulcerative colitis. Int J Mol Med 2004;13:643-647.

34. Faghfoori Z, Shakerhosseini R, Navai L, Somi MH, Nikniaz Z, Abadi A. Effects of an oral supplementation of germinated barley foodstuff on serum CRP level and clinical signs in patients with ulcerative colitis. Health Promot Perspect 2014; 4:116-121.

35. Brotherton CS, Taylor AG, Bourguignon C, Anderson JG. A high-fiber diet may improve bowel function and health-related quality of life in patients with Crohn disease. Gastroenterol Nurs 2014;37:206-216.

36. Marton LT, Goulart RA, Carvalho ACA, Barbalho SM. Omega fatty acids and inflammatory bowel diseases: an overview. Int J Mol Sci 2019;20:4851.

37. Barbalho SM, Goulart Rde A, Quesada K, Bechara MD, de Carvalho Ade C. Inflammatory bowel disease: can omega-3 fatty acids really help? Ann Gastroenterol 2016;29:37-43.

38. Grimstad T, Berge RK, Bohov P, et al. Salmon diet in patients with active ulcerative colitis reduced the simple clinical colitis activity index and increased the anti-inflammatory fatty acid index: a pilot study. Scand J Clin Lab Invest 2011;71:68-73.

39. Scaioli E, Sartini A, Bellanova M, et al. Eicosapentaenoic acid reduces fecal levels of calprotectin and prevents relapse in patients with ulcerative colitis. Clin Gastroenterol Hepatol 2018;16:1268-1275.

40. Yasueda A, Shinzaki S, Iijima H, et al. Safety of emulsifying lipid formulation containing omega-3 polyunsaturated fatty acids for patients with Crohn's disease. Anticancer Res 2016; 36:3753-3759.

41. Lev-Tzion R, Griffiths AM, Leder O, Turner D. Omega 3 fatty acids (fish oil) for maintenance of remission in Crohn's disease. Cochrane Database Syst Rev 2014;(2):CD006320.

42. Eppinga H, Peppelenbosch MP. Worsening of bowel symptoms through diet in patients with inflammatory bowel disease. Inflamm Bowel Dis 2016;22:E6-E7.

43. Limdi JK, Aggarwal D, McLaughlin JT. Dietary practices and beliefs in patients with inflammatory bowel disease. Inflamm Bowel Dis 2016;22:164-170.

44. Jones VA, Dickinson RJ, Workman E, Wilson AJ, Freeman AH, Hunter JO. Crohn's disease: maintenance of remission by diet. Lancet 1985;2:177-180.

45. Ritchie JK, Wadsworth J, Lennard-Jones JE, Rogers E. Controlled multicentre therapeutic trial of an unrefined carbohydrate, fibre rich diet in Crohn's disease. Br Med J (Clin Res Ed) 1987;295:517-520.

46. Schreiner P, Yilmaz B, Rossel JB, et al. Vegetarian or glutenfree diets in patients with inflammatory bowel disease are associated with lower psychological well-being and a different gut microbiota, but no beneficial effects on the course of the disease. United European Gastroenterol J 2019;7:767-781. 
47. Herfarth HH, Martin CF, Sandler RS, Kappelman MD, Long MD. Prevalence of a gluten-free diet and improvement of clinical symptoms in patients with inflammatory bowel diseases. Inflamm Bowel Dis 2014;20:1194-1197.

48. Cohen AB, Lee D, Long MD, et al. Dietary patterns and selfreported associations of diet with symptoms of inflammatory bowel disease. Dig Dis Sci 2013;58:1322-1328.

49. Nolan-Clark D, Tapsell LC, Hu R, Han DY, Ferguson LR. Effects of dairy products on Crohn's disease symptoms are influenced by fat content and disease location but not lactose content or disease activity status in a New Zealand population. J Am Diet Assoc 2011;111:1165-1172.

50. Statovci D, Aguilera M, MacSharry J, Melgar S. The impact of western diet and nutrients on the microbiota and immune response at mucosal interfaces. Front Immunol 2017;8:838.

51. Tasson L, Canova C, Vettorato MG, Savarino E, Zanotti R. Influence of diet on the course of inflammatory bowel disease. Dig Dis Sci 2017;62:2087-2094.

52. Barnes EL, Nestor M, Onyewadume L, de Silva PS, Korzenik JR; DREAM Investigators. High dietary intake of specific fatty acids increases risk of flares in patients with ulcerative colitis in remission during treatment with aminosalicylates. Clin Gastroenterol Hepatol 2017;15:1390-1396.

53. Gibson PR, Shepherd SJ. Evidence-based dietary management of functional gastrointestinal symptoms: The FODMAP approach. J Gastroenterol Hepatol 2010;25:252-258.

54. Prince AC, Myers CE, Joyce T, Irving P, Lomer M, Whelan K. Fermentable carbohydrate restriction (Low FODMAP Diet) in clinical practice improves functional gastrointestinal symptoms in patients with inflammatory bowel disease. Inflamm Bowel Dis 2016;22:1129-1136.

55. Cox SR, Prince AC, Myers CE, et al. Fermentable carbohydrates [FODMAPs] exacerbate functional gastrointestinal symptoms in patients with inflammatory bowel disease: a randomised, double-blind, placebo-controlled, cross-over, rechallenge trial. J Crohns Colitis 2017;11:1420-1429.

56. Aggarwal BB, Sundaram C, Prasad S, Kannappan R. Tocotrienols, the vitamin $\mathrm{E}$ of the 21st century: its potential against cancer and other chronic diseases. Biochem Pharmacol 2010;80:1613-1631.

57. Saw TY, Malik NA, Lim KP, et al. Oral supplementation of tocotrienol-rich fraction alleviates severity of ulcerative colitis in mice. J Nutr Sci Vitaminol (Tokyo) 2019;65:318-327.
58. Zallot C, Quilliot D, Chevaux JB, et al. Dietary beliefs and behavior among inflammatory bowel disease patients. Inflamm Bowel Dis 2013;19:66-72.

59. Hwang C, Ross V, Mahadevan U. Popular exclusionary diets for inflammatory bowel disease: the search for a dietary culprit. Inflamm Bowel Dis 2014;20:732-741.

60. Shepherd SJ, Gibson PR. Nutritional inadequacies of the gluten-free diet in both recently-diagnosed and long-term patients with coeliac disease. J Hum Nutr Diet 2013;26:349-358.

61. Taetzsch A, Das SK, Brown C, Krauss A, Silver RE, Roberts SB. Are gluten-free diets more nutritious? An evaluation of selfselected and recommended gluten-free and gluten-containing dietary patterns. Nutrients 2018;10:1881.

62. Melini V, Melini F. Gluten-free diet: gaps and needs for a healthier diet. Nutrients 2019;11:170.

63. Vernia P, Loizos P, Di Giuseppantonio I, Amore B, Chiappini A, Cannizzaro S. Dietary calcium intake in patients with inflammatory bowel disease. J Crohns Colitis 2014;8:312-317.

64. Szilagyi A, Galiatsatos P, Xue X. Systematic review and metaanalysis of lactose digestion, its impact on intolerance and nutritional effects of dairy food restriction in inflammatory bowel diseases. Nutr J 2016;15:67.

65. Górska-Warsewicz H, Rejman K, Laskowski W, Czeczotko M. Milk and dairy products and their nutritional contribution to the average polish diet. Nutrients 2019;11:1771.

66. Bernstein CN, Fried M, Krabshuis JH, et al. World Gastroenterology Organization practice guidelines for the diagnosis and management of IBD in 2010. Inflamm Bowel Dis 2010; $16: 112-124$

67. Bernstein CN, Eliakim A, Fedail S, et al. World Gastroenterology Organisation global guidelines inflammatory bowel disease: update August 2015. J Clin Gastroenterol 2016;50:803818.

68. Bischoff SC, Escher J, Hébuterne X, et al. ESPEN practical guideline: clinical nutrition in inflammatory bowel disease. Clin Nutr 2020;39:632-653

69. Lee J, Allen R, Ashley S, et al. British Dietetic Association evidence-based guidelines for the dietary management of Crohn's disease in adults. J Hum Nutr Diet 2014;27:207-218.

70. Crohn's and Colitis Foundation of America. Diet and nutrition [Internet]. c2020 [cited 2021 Jun 16]. https://www.crohnscolitisfoundation.org/diet-and-nutrition. 